\title{
CCD astrometric observations of Phoebe in 2003-2004^
}

\author{
R. C. Qiao ${ }^{1,3}$, Z. H. Tang ${ }^{2,3}$, K. X. Shen ${ }^{1}$, G. Dourneau ${ }^{4}$, Y. R. Yan², Y. Yu², S. H. Wang ${ }^{2}$, and J. R. Liu ${ }^{1}$ \\ 1 National Time Service Center, Chinese Academy of Sciences, PO Box 18, Lintong, 710600 Shaanxi, PR China \\ e-mail: rcqiao@ntsc.ac.cn \\ 2 Shanghai Astronomical Observatory (SHAO), Chinese Academy of Sciences, 200030 Shanghai, PR China \\ 3 United Laboratory for Optical Astrometry, Chinese Academy of Sciences, PR China \\ 4 Observatoire Aquitain des Sciences de l'Univers, UMR 5804 Laboratoire d'Astrodynamique, d'Astrophysique et d'Aéronomie de \\ Bordeaux (L3AB), 2 rue de l'Observatoire, 33270 Floirac, France
}

Received 20 December 2005 / Accepted 2 March 2006

\section{ABSTRACT}

\begin{abstract}
In 2003-2004, we obtained 115 new observations of Phoebe, the 9th Saturnian faint satellite (visual magnitude of about 16.5). We used a large CCD detector $(2048 \times 2048$ pixels $)$ mounted on the $1.56 \mathrm{~m}$ astrometric reflector at the Sheshan Station, near Shanghai. In our reduction, an up-to-date catalogue of stars, UCAC2 (Zacharias et al. 2004), was chosen to ensure a proper astrometric calibration. A comparison of our observations to three recently available, high quality ephemerides, including the JPL SAT185 by Jacobson (2004b), has shown that most of our observed positions of Phoebe present an accuracy of some tens of mas, which appears to be a very high level for such a faint satellite.
\end{abstract}

Key words. planets and satellite: general - astrometry

\section{Introduction}

At present, NASA's Cassini mission to explore the Saturnian system, in which a spacecraft will pass close to Phoebe, has begun. However, since its discovery by Pickering (1898) at the end of the nineteenth century, only a few observations of Phoebe, the 9th satellite of Saturn, were made. This is due to its extremely faint visual magnitude (about 16.5) and to its great distance from the primary. Recently, Fienga et al. (2002) pointed out that the quality of the ephemerides will decrease if no new observations are provided in the near future. The situation gives impetus to scientists to acquire new, high quality astrometric observations of this satellite. In recent years, several new series of accurate CCD observations of Phoebe have been published by Veiga et al. (2000), Fienga et al. (2002), and Peng et al. (2004). Veiga et al. (2000) and Fienga et al. (2002) used the very high density USNO A2 star catalogue (Monet et al. 1998), while Peng et al. (2004) used the high accuracy ACT catalogue (Urban et al. 1998) with an overlap reduction. In this paper, we have chosen an alternative method by using the brand new, high density and high accuracy star catalogue UCAC2 (Zacharias et al. 2004) to calibrate our CCD fields. Furthermore, as we benefited from a large CCD cell $(2048 \times 2048$ pixels $)$, many UCAC2 stars were located in each wide CCD field, allowing for a valuable astrometric reduction.

The purpose of this paper is to report our observations of Phoebe, which began in 2003 at the Sheshan Station (E $121^{\circ} 11^{\prime} 03^{\prime \prime} 3$, N 31 $05^{\prime} 46^{\prime \prime} 1$, H $97 \mathrm{~m}$ ) near the Shanghai Astronomical Observatory (SHAO) and to publish our observed data. In December 2003 and in March 2004, two observing missions were carried out on schedule. A total of 115 observations

^ The data are available in electronic form at the CDS via anonymous ftp to cdsarc.u-strasbg. fr or via

http://cdsweb. u-strasbg.fr/cgi-bin/qcat?J/A+A/454/379 obtained from the two missions are presented in this paper. The observation and measurement procedures are described in Sect. 2. Section 3 presents the method of astrometric reduction using the UCAC2 catalogue. In Sect. 4, our observations are compared to the three most recent ephemerides of Phoebe. Also, a comparison of different sets of modern observations, including ours, is made. An analysis of these residuals will follow, to evaluate the accuracy of the observations presented in this paper.

\section{Observation and measurements}

All the observations were made with the $1.56 \mathrm{~m}$ astrometric reflector equipped with a large, new, liquid-nitrogen-cooled CCD chip, $(2048 \times 2048$ pixels, where 1 pixel $=0.024 \mathrm{~mm})$ at the Sheshan Station of SHAO. When it was necessary to get a higher signal-to-noise ratio during the observation, the binning mode was used, so that the charges of $2 \times 2$ pixels could be read out together. Consequently, the pixel number became $1024 \times 1024$, and the size of each pixel was $0.048 \mathrm{~mm}$. The exposure times varied from $30 \mathrm{~s}$ to $180 \mathrm{~s}$, depending on the meteorological conditions. The reflector has a focal length of $15.6 \mathrm{~m}$ and the total size of the CCD is about $50 \times 50 \mathrm{~mm}$, corresponding to a field of about $11^{\prime} \times 11^{\prime}$. For more instrumental details concerning the CCD detector and reflector, see Qian \& Tao (2003).

A total of 115 frames were obtained over 9 nights. $101 \mathrm{im}$ ages were made in 6 nights in December 2003 and 14 images in 3 nights in March 2004. In our previous observations, the centroid of all the satellites was measured manually by using the Image Reduction and Analysis Facility (IRAF) center finding tool. But in the present work, because of the use of a CCD detector with a wider field than the CCD used in previous years, the objects to be measured included a much larger number of background stars available in the field. Thus, centering became very laborious and time-consuming. Consequently, an automatic 


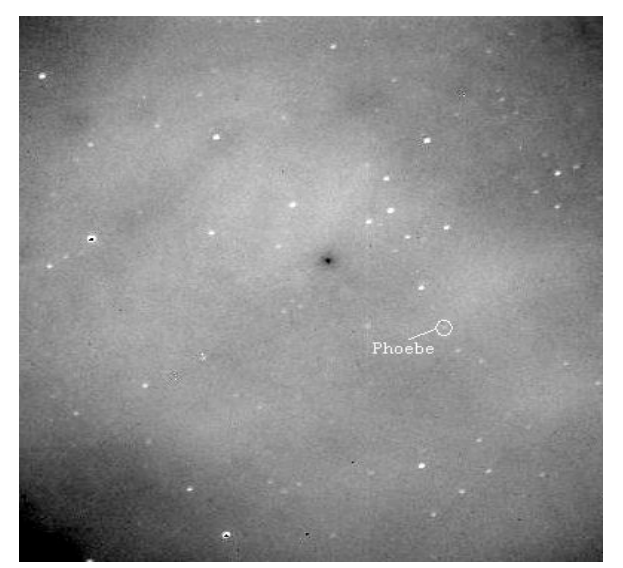

Fig. 1. CCD frame observed $1 / 12 / 2003$, at $15^{\mathrm{h}} 02^{\mathrm{mn}}$ UTC. The exposure time is $2^{\mathrm{mn}}$, and the CCD dimensions are $1024 \times 1024$ square pixels.

detection and measurement of the objects appeared to be necessary. For that reason, we adopted a measurement-centering procedure in which all the target images were carried out in batch mode. We used the "DAOFIND" task from the IRAF software package for photometric measurement. The detailed procedure used is as follows:

- 1. After extracting several typical CCD images from the observations each night, the Full Width at Half-Maximum (FWHM) was found for each image. Then, the FWHM average values were calculated to be between 2.5 and 4 pixels.

- 2. The rough sky background bias was estimated and removed from each image. The seeing level at the Sheshan Station is 1.1-1.9 arcsec, typically 1.5 arcsec. The FWHM of images is larger than seeing level because of the guide and focus errors.

- 3. The "DAOFIND" command was used in batch mode to scan all the CCD images for a preliminary location of all the targets.

- 4. The "CENTER" command was used in batch mode to accurately determine the positions of all the targets. This command proposes three methods for computing the center of images: CENTROID, GAUSS, and OFILTER. We adopted the Gaussian method, which uses a 2-dimensional Gaussian function, including a term to represent the background level, to fit the intensity of image. However, for a small number of images, the positions of objects still had to be manually measured because of the extreme faintness of Phoebe.

In Fig. 1, a typical CCD frame with Phoebe is presented to exemplify the observing and measurement procedures. In the frame, Phoebe is marked by a circle.

\section{Astrometric reduction}

Because of the great distance between Phoebe and Saturn, no major Saturnian satellite appears in the same CCD frame as Phoebe. Thus, the differential measurement relative to the major satellites of Saturn appears to be inconvenient. Also, an astrometric reduction from the theoretical positions of Saturn's inner satellites cannot be performed, as it could be in previous works (Shen et al. 2001). To overcome this difficulty, we had the opportunity to benefit from two important advantages in this work. First of all, the large CCD chip $(2048 \times 2048$ pixels $)$ corresponds to a wide field of about $11^{\prime} \times 11^{\prime}$. Thus, the number of stars present in each CCD field is large enough to allow us to carry
Table 1. Extract of the list of our observed positions of Phoebe available on request at the CDS. These positions are topocentric and given in the ICRF J2000 system.

\begin{tabular}{ccccc}
\hline \hline Year & M & Day(UTC) & RA(J2000) & Dec(J2000) \\
\hline 2003 & 12 & 1.62639 & $6^{\mathrm{h}} 49^{\mathrm{m}} 8.4058$ & $+22^{\circ} 10^{\prime} 25^{\prime \prime} \cdot 395$ \\
2003 & 12 & 1.62917 & $6^{\mathrm{h}} 49^{\mathrm{m}} 8.3587$ & $+22^{\circ} 10^{\prime} 25^{\prime \prime} 426$ \\
2003 & 12 & 1.63194 & $6^{\mathrm{h}} 49^{\mathrm{m}} 8.3114$ & $+22^{\circ} 10^{\prime} 25^{\prime \prime} \cdot 742$ \\
2003 & 12 & 1.63472 & $6^{\mathrm{h}} 49^{\mathrm{m}} 8^{\mathrm{s}} .2716$ & $+22^{\circ} 10^{\prime} 25^{\prime \prime} 470$ \\
2003 & 12 & 1.63819 & $6^{\mathrm{h}} 49^{\mathrm{m}} 8.2156$ & $+22^{\circ} 10^{\prime} 25^{\prime \prime} .583$ \\
2003 & 12 & 1.70694 & $6^{\mathrm{h}} 49^{\mathrm{m}} 7^{\mathrm{s}} .0967$ & $+22^{\circ} 10^{\prime} 27^{\prime \prime} .018$ \\
\hline
\end{tabular}

out a classical astrometric reduction, using the positions of these background stars. The second advantage was that we were able to use the star positions in the very high accuracy UCAC2 star catalogue, since a sufficient number of UCAC2 stars appeared in each wide CCD field.

Zacharias et al. (2004) present the UCAC2 as a very high density catalogue. It contains more than 48 million stars with magnitudes ranging from about 7.5 to 16 and covers the sky from -90 to +40 (or +52 in some areas) degrees in declination. This high density ensures that about 15-20 UCAC2 reference stars are available for each of our CCD images, covering a field of $11^{\prime} \times 11^{\prime}$.

Furthermore, the UCAC2 appears to be one of the best highaccuracy positional star catalogues available today (Zacharias et al. 2004). Its nominal errors in positions are about $0 . ' 02$ for the stars in the 10 to 14 mag range and about 0 .' $^{\prime} 07$ for the limited magnitude of 16. Positions and proper motions are referred to using the International Celestial Reference System (ICRS) and are given at the epoch J2000.0. No significant zonal error is mentioned.

We have seen above that there are enough UCAC2 stars in the field of our CCD frames to carry out the astrometric reduction. Therefore, neither secondary star catalogues of low quality, nor theoretical satellite positions, which could bias the astrometric reduction by giving preference to the theory used as a reference (Shen et al. 2001), is necessary. So, the use of only the UCAC2 catalogue, with its high quality specifications, should greatly increase the level of accuracy of the derived observed positions of Phoebe presented in this paper.

For more accuracy in the derived observed positions, the reduction process takes into account the proper motions that were applied to the reference stars to carry them forward to the epochs of observations. Then, the typical 6 constants model of plate reduction was selected, according to the procedure previously described by Tang (2002).

All our observations of Phoebe are presented in a catalogue available on request at the CDS, via anonymous ftp to cdsarc.ustrasbg.fr. In Table 1, we give an extract of this catalogue presenting the topocentric mean equatorial astrometric coordinates $(\alpha, \delta)$ of Phoebe, with reference to the ICRF J2000 system. All significant astrometric corrections have been made, including atmosphere differential refraction.

\section{The theoretical model}

An accurate ephemeris is crucial for analyzing the quality of observations. Thus, three different, recent, accurate ephemerides have been used to produce the theoretical positions of Phoebe to compare it to our observations. These ephemerides are described in the following subsections. 


\subsection{JPL ephemeris}

The first ephemeris can be found directly in the JPL's Horizon's system. Phoebe's positions on this site are derived from the newest SAT185 satellite ephemeris by Jacobson (2004b). As with all other recent ephemerides of the major Saturnian satellites developed by Jacobson (2004a), the SAT185 ephemeris of Phoebe is generated by numerically fitting the integrated orbit of this satellite to Earth-based observations from 1966 to 2003 and to data obtained with spacecrafts including Pioneer 11, Voyager, and more recently, Cassini. The accuracy of the SAT185 theoretical positions of Phoebe is claimed to be $0{ }^{\prime} 13$ in $\alpha$ and in $\delta$.

The planetary ephemeris used is DE405, which has replaced DE200 since 2003. The accuracy of the position of DE405 has been significantly improved with the use of enhanced observational data, including spacecraft range and doppler measurements (Standish 2004). DE405 appears to be one of the most accurate planetary ephemerides available now, as it presents an accuracy of about $1 \mathrm{~km}$ for inner planets and about 0.1 arcsec for outer planets such as Saturn.

\subsection{The orbit of Phoebe by Shen et al. (2005)}

The second ephemeris that we used to compare our observations to is derived from the orbit recently re-determined by Shen et al. (2005). It was obtained from a numerically integrated fit of the motion of Phoebe to a large set of old ground-based observations spanning the period 1906 to 1995 , together with recent high-quality post-1995 CCD observations. The collection of recent CCD observations used to fit this orbit includes the observations by Veiga et al. (2000), Fienga et al. (2002), and Peng et al. (2004), as well as the observations from December 2003 presented in this paper.

The calculation of this orbit is based on an integration program from Harper (1993), which is a 12-order Runge-Kutta-Nystrom numerical integration with a variable step size. The calculation of perturbations includes the effects of the Sun, Jupiter, Uranus, and Titan. The perturbations from the Sun, Jupiter, and Uranus are computed by using their positions derived from the planetary ephemeris DE405. A complete analytical model, which is similar to those previously used by Taylor \& Shen (1988) and by Harper \& Taylor (2003), is employed for computing the perturbations by Titan. Shen et al. (2005) have evaluated the accuracy of their new orbit to about 90 mas for the recent period.

\subsection{A model for Phoebe from the IMCCE}

The third ephemeris of Phoebe used for the comparison of our observations was chosen from the two provided by the IMCCE web sites. The first of these ephemerides is derived from Bec-Borsenberger \& Rocher (1982). We preferred to use the second ephemeris, recently improved by Arlot et al. (2003) by using much more recent observations. This ephemeris, which has already been slightly improved since its publication, is available via the IMCCE web site: http://www. imcce.fr/hosted_sites/saimirror/nssephhf.htm/.

This orbit has been fitted to the same Earth-based astrometric observations that Shen et al. (2005) used, excluding our December 2003 observations presented in this paper. Therefore, both of these orbits should have a comparable accuracy. Arlot et al. (2003) give standard deviations to the mean of about $0 . ' 18$ for recent observations, which should be an approximate value of the contemporary accuracy of their orbit.
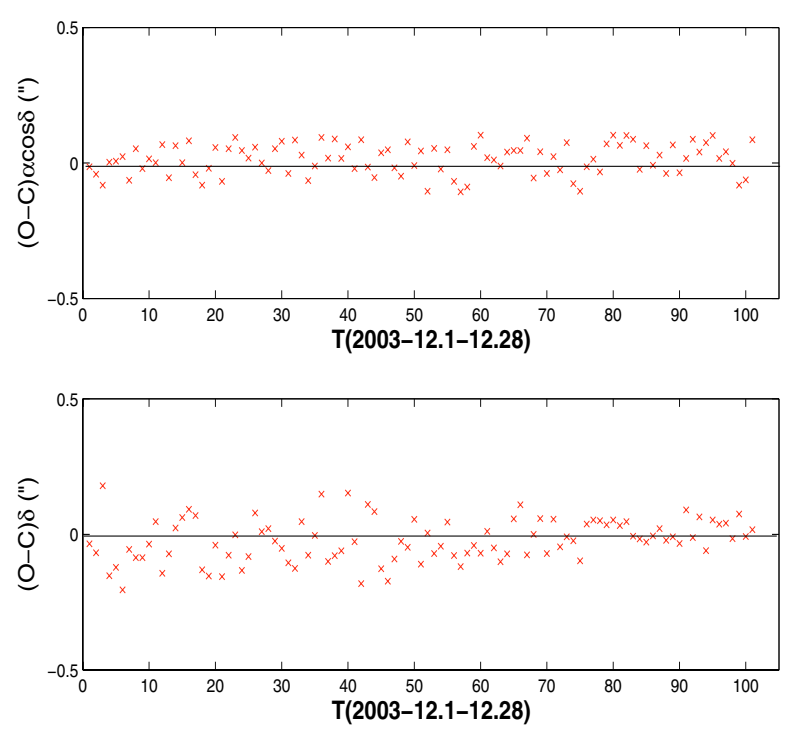

Fig. 2. Plots of residuals in right ascension and declination of the absolute positions of Phoebe, derived from the comparison of our 2003 observations to the JPL ephemeris vs. time $T(1 / 12 / 2003-28 / 12 / 2003)$.
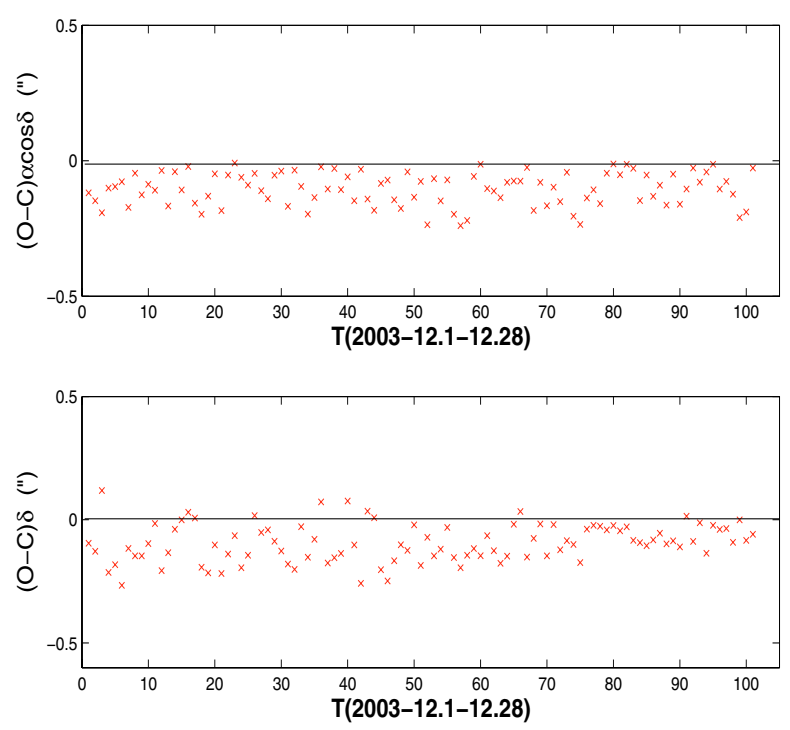

Fig. 3. Plots of residuals in right ascension and declination of the absolute positions of Phoebe, derived from the comparison of our 2003 observations to the SHN ephemeris vs. time $T(1 / 12 / 2003-28 / 12 / 2003)$.

\section{Comparison with theoretical positions}

Our two sets of observations made in December 2003 and in March 2004 have been compared to the theoretical positions derived from the three models presented above. The obtained residuals $(\mathrm{O}-\mathrm{C})$ larger than $2^{\prime \prime}$ have been rejected. Figures 2-4 plot the residuals $(\mathrm{O}-\mathrm{C})$ versus time for the observations made in December 2003. They display a rather low dispersion of the dots, as well as a good agreement of the observations with all three theories.

To quantify the accuracy of our observations and their agreement to the theories, we computed the mean residuals $\mu$ and the standard deviations to the mean $\sigma$ of the absolute positions of Phoebe that are given in Table 2.

First, the analysis of standard deviations to the mean can provide some information about the accuracy of the observations. 

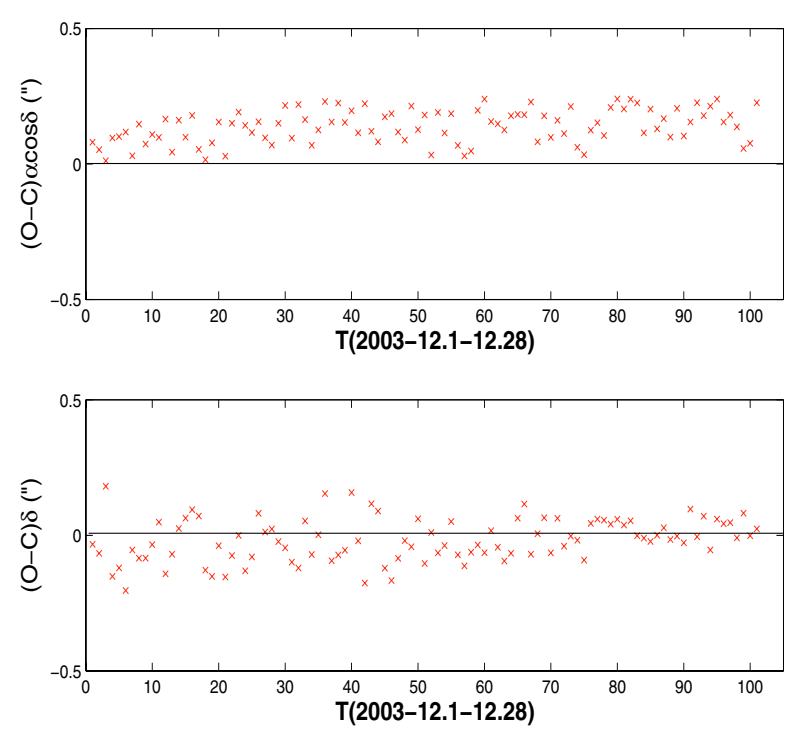

Fig. 4. Plots of residuals in right ascension and declination of the absolute positions of Phoebe, derived from the comparison of our $2003 \mathrm{ob}-$ servations to the IMCCE ephemeris vs. time $T(1 / 12 / 2003-28 / 12 / 2003)$.

Table 2. Mean residuals $\mu\left({ }^{\prime \prime}\right)$ and standard deviations to the mean $\sigma\left({ }^{\prime \prime}\right)$ of the absolute positions of Phoebe for the 2 sets of observations made in December 2003 and in March 2004. These observations are compared to three recent ephemeris: JPL (Jacobson 2004), SHN (Shen et al. 2005), and IMCCE (Arlot et al. 2003). $N_{\mathrm{u}}$ is the number of observed positions for each set of observations.

\begin{tabular}{lcccccccc}
\hline \hline Set & & \multirow{2}{*}{$N_{\mathrm{u}}$} & \multicolumn{2}{c}{ JPL } & \multicolumn{2}{c}{ SHN } & \multicolumn{2}{c}{ IMCCE } \\
& & & $\sigma$ & $\mu$ & $\sigma$ & $\mu$ & $\sigma$ & $\mu$ \\
\hline 2003 & $\Delta \alpha \cos \delta$ & 101 & 0.058 & 0.014 & 0.056 & -0.096 & 0.061 & 0.139 \\
& $\Delta \delta$ & 101 & 0.078 & -0.023 & 0.077 & -0.094 & 0.078 & -0.018 \\
\hline 2004 & $\Delta \alpha \cos \delta$ & 14 & 0.257 & 0.103 & 0.261 & 0.631 & 0.257 & 0.304 \\
$\Delta \delta$ & 14 & 0.376 & -0.004 & 0.381 & -0.010 & 0.376 & 0.011 \\
\hline
\end{tabular}

For a same set of observations, Table 2 shows that the values of the standard deviations to the mean $\sigma$ are very close, regardless of the ephemeris used. The analysis of the values of $\sigma$ shows that the observations made in December 2003 present a very high accuracy of some tens of mas. In contrast, the observations made in March 2004 appear to be significantly worse, with an accuracy of some hundreds of mas. This is likely due to the poor weather conditions during this observation period, leading to CCD fields with a small number of reference stars and to images of the faint satellite Phoebe that were very difficult to measure.

Also, analysing the mean residuals $\mu$ can provide information about the agreement of the different ephemeris with our observations. Table 2 shows the very low mean residuals, generally under 20 mas, obtained for the JPL ephemeris of Jacobson (2004b). The values obtained for the two other ephemerides are significantly higher, about 0 .' 1 for the $2003 \mathrm{ob}-$ servations, and more for the 2004 observations, which present a lower quality, due to bad weather conditions.

This difference of accuracy between the very high quality JPL SAT185 ephemeris of Phoebe by Jacobson (2004b) and the other ephemerides can be explained in that the JPL ephemeris has been derived from only recent observations, including new high-accuracy spacecraft data, while both of the other ephemerides have been fitted to only Earth-based observations,
Table 3. Mean residuals $\mu\left({ }^{\prime \prime}\right)$ and standard deviations to the mean $\sigma\left({ }^{\prime \prime}\right)$, given in right ascension and declination, for the latest sets of observations. These sets include our observations made in December 2003, as well as previous observations by Veiga et al. (2000), Fienga et al. (2002), and Peng et al. (2004). All observations are compared to the JPL ephemeris. $N_{\mathrm{u}}$ is the number of the observed positions of Phoebe for each set of observations.

\begin{tabular}{cccccc}
\hline \hline Author & $N_{\mathrm{u}}$ & $\sigma_{\alpha}$ & $\mu_{\alpha}$ & $\sigma_{\delta}$ & $\mu_{\delta}$ \\
\hline Veiga & 60 & 0.140 & -0.080 & 0.260 & 0.290 \\
Fienga & 163 & 0.148 & 0.156 & 0.177 & 0.154 \\
Peng & 50 & 0.068 & -0.011 & 0.061 & -0.073 \\
This paper & 101 & 0.058 & 0.014 & 0.078 & -0.023 \\
\hline
\end{tabular}

including large sets of old, rather inaccurate, photographic observations (see Sect. 4).

To compare the accuracy of our observations to previous ones, we present the residuals of several recent sets of CCD observations of Phoebe, including our observations of December 2003 and the observations made by Veiga et al. (2000), Fienga et al. (2002), and Peng et al. (2004), in Table 3.

The analysis of the standard deviations to the mean $\sigma$ given in Table 3 shows that our observations have a significantly better accuracy, with values of about 0.07 , than previous observations by Veiga et al. (2000) and by Fienga et al. (2002), which present greater $\sigma$ values, up to 0.26 . This is likely due to the use, in the present work, of the UCAC2 star catalogue, which is significantly more accurate than the USNO A2 (Monet et al. 1998) used in both of these previous works. Also, we can observe that our observations have reached the high accuracy of the most recent observations by Peng et al. (2004), who used the ACT catalogue (Urban et al. 1998) with an overlapping reduction method.

It is likely that this result occurs because, unlike Peng et al. (2004), we benefited from a large CCD field and the UCAC2 catalogue.

The analysis of the mean residuals of Table 3 enhances the level of accuracy of the JPL ephemeris. All the values of these residuals are lower than 0.15 , except in declination for the observations of Veiga et al. (2000), which are not the most accurate ones. Moreover, these mean residuals remain below 0.'07 for both of the most recent sets of observations by Peng et al. (2004) and by the authors of this paper. Therefore, this confirms that the JPL ephemeris of Phoebe should really present an accuracy better than about 0 .'13, as claimed on the Horizon system.

\section{Conclusion}

In this paper, we presented the 115 astrometrically observed positions of Phoebe that we made in December 2003 and in March 2004 on the $1.56 \mathrm{~m}$ reflector at the Sheshan Station, near the Shanghai Observatory. We compared our observations to three recent ephemerides by Jacobson (2004b), Shen et al. (2005), and Arlot et al. (2003). First, we confirmed the high level of accuracy of the JPL ephemeris by Jacobson (2004b), giving positions with errors generally lower than $0 .{ }^{\prime} 1$, due to their fit to recent accurate observations, including spacecraft data.

Also, this comparison has shown the high accuracy of our observations, to some tenths of arcseconds, for the 101 positions observed in 2003. The 14 other observed positions obtained in 2004 appear significantly less accurate, due to the bad weather conditions of observation. The high level of accuracy of our observations made in 2003 is likely due to the following elements. First, we used a large field CCD detector with $2048 \times 2048$ pixels 
corresponding to $11^{\prime} \times 11^{\prime}$. Then, the UCAC2 high density catalogue allowed us to employ an astrometric reduction from the stars of this accurate catalogue, since a great number of them appeared on each CCD frame. This has led us to a significantly higher accuracy than all previous observations of Phoebe and to a level of accuracy equivalent to the most recent ones by Peng et al. (2004).

In a future work, we would like to make an attempt to again improve the accuracy of the observed positions of this satellite. A more accurate catalogue than UCAC2, such as Tycho2 (Hog et al. 2000) or ACT (Urban et al. 1998), could be used. But these catalogues present a lower density than UCAC2. Consequently, the limited number of catalogued stars used in the astrometric reduction could reduce its accuracy. Furthermore, in the special case of Phoebe, the very faint 9th satellite of Saturn, the measurement of its images appears to be rather difficult. This could also limit any future attempt for improving the high level of accuracy already obtained in the present work.

Nonetheless, we plan to carry on observing Phoebe over the next three years and to make some new attempts to improve the observed positions, as discussed above. This will increase the density of the positional data of Phoebe and should contribute to significant future improvements in the caculation of Phoebe's orbit.

Acknowledgements. The authors are very grateful to the staff at the Sheshan Station of the Shanghai Observatory for their assistance, and especially to
Dr. J. Tao for providing us with much assistance throughout our observing run. We also would like to express our sincere thanks to Dr. Harper for his valuable help, in particular, for providing his integration program. This work was carried out with financial support from the National Science Foundation of China (NSFC) (Grant Nos. 10273015, 10573018).

\section{References}

Arlot, J.-E., Borsenberger, A., Fienga, A., et al. 2003, A\&A, 411, 309

Bec-Borsenberger, A., \& Rocher, P. 1982, A\&AS, 50, 423

Fienga, A., Arlot, J.-E., Baron, N., et al. 2002, A\&A, 391, 767

Harper, D. 2003, private communication

Harper, D., \& Taylor, D. B. 1993, A\&A, 268, 326

Hog, E., Fabricius, C., Makarov, V. V., et al. 2000, A\&A, 363, 385

Jacobson, R. A. 2004a, AJ, 128, 492

Jacobson, R. A. 2004b, SAT185, JPL satellite ephemeris

Monet, D., Bird, A., Canzian, B., et al. 1998, The USNO-A2.0 Catalogue, U.S. Naval Observatory

Peng, Q. Y., Vienne, A., Shen, K. X., Han, Y. B., Li, Z. L. 2004, A\&A, 424, 339

Pickering, E. C. 1898, Harv. Coll. Obs. Circ., 43

Qian, B. C., \& Tao, J. 2003, PASP, 115, 490

Shen, K. X., Dourneau, G., Qiao, R. C., et al. 2001, A\&A, 367, 1061

Shen, K. X., Harper, D., Qiao, R. C., et al. 2005, A\&A, 437, 1109

Standish, E. M. 2004, A\&A, 417, 1165

Tang, Z. H., Wang, S. H., \& Jin, W. J. 2002, AJ, 123, 125

Taylor, D. B., \& Shen, K. X. 1988, A\&A, 200, 269

Urban, S. E., Corbin, T. E., \& Wycoff, G. L. 1998, AJ, 115, 2161

Veiga, C. H., Vieira Martins, R., \& Andrei, A. H. 2000, A\&AS, 142, 81

Zacharias, N., Urban, S. E., Zacharias, M. I., et al. 2004, AJ, 127, 3043 